\title{
Primary mucin-secreting sweat gland carcinoma of the eyelid: a case report and review of literature
}

\begin{abstract}
A 55 year-old man presented with a history of gradually enlarging mass in the left lower eyelid. Patient had no tumor elsewhere in body. The mass was surgically excised and histopathology was performed. Histopathology revealed mucinous carcinoma of eyelid. No evidence of recurrence or metastasis was noted till date postoperatively. Rarity of primary mucinous carcinoma involving the eyelid prompted us to report the case.
\end{abstract}

Keywords: mucinous carcinoma, eyelid, primary

\author{
Volume 3 Issue 2 - 2018
}

\author{
Khairwa A,' Sechdeva $S,{ }^{2}$ Chug A, ${ }^{2}$ Kumar $G^{3}$ \\ 'Department of Pathology, ESIC Model Hospiatal, India \\ ${ }^{2}$ Department of Pathology, Holy Family hospital, India \\ ${ }^{3}$ Department of Ophthalmology, Holy Family hospital, India
}

Correspondence: Anju Khairwa, Department of Pathology, ESIC Model Hospiatal, FTA, 504,AV Nagar, New Delhi, India, Tel: +919810436138, Email anjukhairwa@gmail.com

Received: May 05, 2017 | Published: May 18, 2018

\section{Introduction}

Primary cutaneous mucinous carcinoma is a rare, low-grade malignant tumor arising preferentially on the head and neck, especially in the eyelid. ${ }^{1}$ Mucinous tumor has been reported under many different names, including adenocystic, colloid, gelatinous, and mucinous eccrine carcinoma. The latter name is derived in part from older studies that suggested features of eccrine secretory epithelium within the neoplastic component. ${ }^{2,3}$ This tumor is mostly diagnosed as a metastasis tumor; rarely as primary. The clinical diagnosis of primary mucinous carcinoma of eyelid may be difficult because this tumor does not have a pathognomonic clinical appearance, and is always asymptomatic. This tumor may present as a painless, slow-growing mass and is usually ignored by the patient and wrongly diagnosed by clinician. The vital treatment for primary mucinous carcinoma is complete surgical excision. We report a patient who presented with primary mucinous carcinoma of the eyelid.

\section{Case report}

A 55 year old male presented with a swelling of left lower eyelid for last 10 months. The lesion increased in size very slowly and it was associated with mild tenderness and itching. The patient was known case of diabetes mellitus on treatment. Patient has no history of fever, cough, and hypertension. There was no mass lesion elsewhere in body. Physical examination revealed a $1 \times 1 \mathrm{~cm}$, mild tender, soft to firm and cystic lesion situated over the left lower eyelid. The clinical diagnosis was epidermal inclusion cyst. The mass was then completely excised and sent for histopathological examination. Grossly, the lesion was well circumscribed, covered by skin, and measuring $1 \times 1 \times 0.5 \mathrm{~cm}$. The cut surface of it was grey-white and gelatinous. Histopathological examination of the lesion showed characteristic appearance of mucinous carcinoma supports primary malignancy (Figure 1A1C). The tumor was seen below epidermis in intradermal location and was well circumscribed. The tumor was divided into numerous compartments by strands of delicate fibrous septa. Each compartment showed an abundance pool of slightly basophilic mucin. Within the mucin pool, small cluster and island of tumor cells were floating. The tumor cells were arranged in nests or cords and cribriform pattern. The tumor cells showed slight nuclear pleomorphism, round to oval nuclei and abundant pale to eosinophilic cytoplasm. Few mitotic figures were also noted. Overlying epidermis was unremarkable. These tumor cells were positive for mucicarmine, periodic acid Schiff (PAS), PAS with diastase resistant and Alcian blue (pH2.5) stains. Whole body PET scan did not reveal any tumor focus in body. Therefore, final diagnosis of primary mucinous carcinoma of eyelid was made.

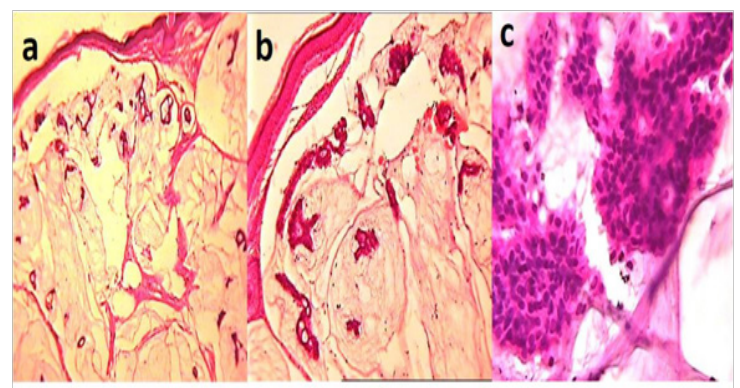

Figure 1: A panel of microphotographs of Primary cutaneous mucinous carcinoma; (A) Section showing intra-dermal located well circumscribed tumor with pool of mucin and floated clusters of tumor cells (H\&E, 4X). (B) Section showing islands of neoplastic basaloid cells ranging from nests or cords to cribriform arrangements within mucinous pools partitioned by thin fibrous septa (H\&E, 20X). (C) The tumor cells showed slight nuclear pleomorphism, round to oval nuclei and abundant pale to eosinophilic cytoplasm and few mitotic figures (H\&E, 40X).

\section{Discussion}

Primary mucinous carcinoma of skin was first reported by Lotzbeck in $1859 .{ }^{4}$ A histogenetic link between primary cutaneous mucinous carcinoma and sweat gland was recognized and accepted in the 1950s. ${ }^{5}$ Currently classified by the World Health Organization under the category of malignant tumors with apocrine and eccrine differentiation. ${ }^{1}$ Few studies described that these tumors were originally derived from eccrine coil epithelium. ${ }^{3}$ But recently, these were referred as mucinous eccrine carcinoma. ${ }^{6}$ Wright and Font published largest study of mucinous carcinoma of the eyelid (21 cases). ${ }^{3}$ Clinically, primary cutaneous mucinous carcinomas of the eyelid are slow-growing tumors with a duration of several months to several years, usually occurring in more elderly individuals (average age 62.6 years, range $8-87$ years). ${ }^{6}$ These lesions have been described 
as painless, papular or nodular, with sizes ranging from $5 \mathrm{~mm}$ to 120 $\mathrm{mm} .^{7}$ They are frequently single, isolated lesions with color reported as red, pink, gray, blue or purple. ${ }^{7,8}$ In present case had characteristic clinical features. In our case, the lesion clinically was diagnosed as epidermal inclusion cyst. In other studies the lesions were clinically diagnosed as basal cell carcinoma, keratoacanthoma, nevus, or apocrine hidrocystoma. ${ }^{8}$ The tumor contains islands of neoplastic basaloid cells having solid to cribriform arrangements within slightly basophilic, periodic acid-Schiff-positive mucinous pools, sometimes partitioned by thin fibrous septa. ${ }^{1}$ The mucin is Alcian blue positive $(\mathrm{pH} 2.5)$ and stains with mucicarmine and colloidal iron. According Mendoza et al., ${ }^{8}$ as a sialomucin, it is hyaluronidase resistant and sialidaselabile. ${ }^{8}$ Cytologically, the tumor cells are characteristically round to cuboidal with moderate amounts of cytoplasm and typically with a low mitotic count and little nuclear atypia. ${ }^{1}$ Same histopathological findings were noted in our case. Usually, mucinous carcinoma of eyelid is metastatic from another site. Mucinous carcinoma arises most commonly from the breast, and gastrointestinal or respiratory tract, but may also originate from salivary and lacrimal glands, urinary tract, prostate, or paranasal sinuses. In our case, we excluded primary mucinous carcinoma elsewhere in body by whole body PET scan. Primary mucinous carcinoma of the skin typically has an indolent course. Local recurrence occurs frequently $(29.4 \%)$ following excision, but the rate of metastasis is low (9.6\%). Most metastases are to regional lymph nodes, although widespread metastases have been reported. ${ }^{3}$ There is no recurrence in our case till now. Treatment for primary mucinous carcinoma of the skin is wide local excision. Weber et al first reported the use of Mohs micrographic surgery for the treatment of primary mucinous carcinoma of the eyelid. ${ }^{9}$

\section{Conclusion}

Primary cutaneous mucinous carcinoma of the eyelid is an uncommon variant of sweat gland tumor with an indolent course and infrequent metastasis relative to true sweat gland tumors. Treatment includes wide local excision and lymph node dissection in the setting of clinically positive nodes. Because of frequent local recurrences, close follow-up is warranted.

\section{Acknowledgements}

None.

\section{Conflict of interest}

Authors declare that there is no conflict of interest.

\section{References}

1. Le Boit PE, Burg G, Weedon D, et al. Pathology and Genetics of Skin Tumors. Lyon, France: IARC Press; 2006.

2. Headington JT. Primary mucinous carcinoma of skin: histochemistry and electronmicroscopy. Cancer. 1977;39:1055-1063.

3. Wright JD, Font RL. Mucinous sweat gland adenocarcinoma of eyelid: a clinic-pathologic study of 21 cases with histochemical and electron microscopicobservations. Cancer. 1979;44:1757-1768.

4. Smith CC. Metastasizing carcinoma of the sweat-glands. $\mathrm{Br} J$ Surg. 1955;43:80-84.

5. Lennox B, Pearse AG, Richards HG. Mucin-secreting tumours of the skin with special reference to the so-called mixed-salivary tumour of the skin and its relationto hidradenoma. J Pathol Bacteriol. 1952;64:865-880.

6. Liszauer $\mathrm{AD}$, Brownstein $\mathrm{S}$, Codère $\mathrm{F}$. Mucinous eccrine sweat gland adenocarcinomaof the eyelid. Can J Ophthalmol 1988;23:17-21.

7. Snow SN, Reizner GT. Mucinous eccrine carcinoma of the eyelid. Cancer. 1992;70:2099-2104.

8. Mendoza S, Helwig EB. Mucinous (adenocystic) carcinoma of the skin. Archives of Dermatology. 1971;103:68-78.

9. Weber PJ, Hevia O, Gretzula JC, et al. Primary mucinous carcinoma. $J$ Dermatol Surg Oncol. 1988;14:170-172. 